\title{
Elastic contact of a stiff thin layer and a half-space
}

\author{
J. Kaplunov, D. Prikazchikov, L. Sultanova
}

\begin{abstract}
The 3D problem in linear elasticity for a layer lying on a half-space is subject to a two-parametric asymptotic treatment using the small parameters corresponding to the relative thickness of the layer and stiffness of the foundation. General scaling for the displacements and stresses is inspired by the analysis of the exact solution of the toy plane strain problem for a vertical sinusoidal load. The direct asymptotic procedure widely used in mechanics of thin structures is adapted for the layer. It is demonstrated that the Kirchhoff theory for thin plates is only applicable for sufficiently high contrast of the coating and half-space stiffnesses. In the scenario, in which the Kirchhoff theory fails, alternative approximate formulations are introduced, reducing the original problem for a coated solid to problems for a homogeneous half-space with Neumann, mixed or effective boundary conditions along its surface.
\end{abstract}

Keywords. Stiff thin coating, Asymptotic, Kirchhoff plate, Contrast, Substrate.

\section{Introduction}

The problem of elastostatics for a stiff layer lying on a half-space is of long term interest for numerous engineering applications. In particular, a variety of problems in structural mechanics are modeled by a thin Kirchhoff plate resting on an elastic substrate, e.g. see [11, 17, 18, 19, 20, 25]. In addition, we cite an influential paper [7] and also a useful servey of mathematical techniques for treating plates on a linear elastic foundation in [26]. At the same time, the range of validity of this approximate formulation, restricted only to thin and sufficiently stiff layers, has not always been fully appreciated. Only very few papers, e.g. [8], explicitly take into account the limitation associated with a specific asymptotic ratio of plate and substrate stiffnesses. The effect of high contrast in stiffness was briefly addressed in [3], studying a contact problem for a coated half-space, without relating it to the relative thickness of the coating. In addition, we refer to [4], appreciating the importance of high contrast limit. Among modern considerations on the subject we also mention [5] inspired by modelling of advance resonant devices, and $[6,9,12,14,15,27]$ tackling a variety of vibration and stability phenomena. In the recent paper [24] a 3D problem in linear elasticity for a soft layer attached to a substrate was treated for a broad range of ratios between relative stiffnesses and wavelengths, resulting, in particular, in the justification and refinement of Winkler-Fuss hypothesis.

In this paper, we extend the methodology in [24] to a complementary scenario of a stiff layer. We operate with two small parameters, corresponding to the relatively small stiffness of the substrate and thickness of the layer, adapting the asymptotic procedure traditional to thin elastic structures, e.g. see $[2,10,13,16]$. Initially, it was developed for Neumann boundary value problems for plates and shells assuming prescribed stresses along the faces. Later on, the procedure was extended to Dirichlet and various mixed boundary value problems, enabling modeling of clamped faces, characteristic of thin coatings. For both setups, the displacements and stresses are expanded into series in the thickness variable, resulting in the $3 \mathrm{D}$ to $2 \mathrm{D}$ dimension reduction. The peculiarity of the studied configuration 
is that the contact with the substrate results in asymptotic consideration of a pretty sophisticated boundary value problem for the thin layer.

The limiting forms of the original elasticity equations for various ratios between these two parameters are derived. First, we establish the asymptotic behaviour of the displacement and stress components for a toy plane strain problem for a vertical sinusoidal load. Next, we adapt the initial settings coming from the toy problem to the general 3D setup. In particular, we confirm the scaling in [8] corresponding to a coupled problem for a Kirchhoff plate resting on an elastic half-space. As might be expected, for a softer substrate we arrive at leading order at an uncoupled problem for plate bending. At the same time, for a stiffer substrate, which is still much softer than the layer, any plate bending theory fails. In the latter case, however, we formulate a set of boundary value problems for a homogeneous half-space. Among them, in particular, there are effective boundary conditions originally derived in [28] and later justified in [10]. The validity of all the shortened approximate formulations is justified by comparison with the exact solution of the aforementioned plane strain problem.

\section{Problem statement}

As in [24], we consider a coated elastic half-space, subject to action of a vertical load $P=P\left(x_{1}, x_{2}\right)$ at the upper face of the layer, see Figure 1.

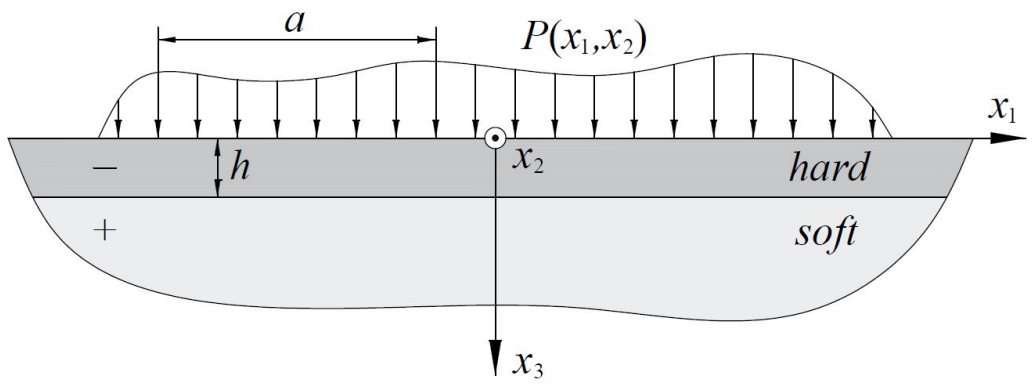

FiguRE 1. Problem statement.

We assume that the thickness of the layer $h$ is small compared to a typical length scale $a$ related to variation of the load along the coordinates $x_{1}, x_{2}$. Therefore, we introduce a small geometrical parameter

$$
\varepsilon=\frac{h}{a} \ll 1
$$

We also suppose that the layer is much stiffer than the half-space, leading to a small material parameter

$$
\mu=\frac{\mu^{+}}{\mu^{-}} \ll 1
$$

where $\mu^{ \pm}$are shear moduli, with " -" and "+" corresponding to the layer and the half-space, respectively. The small parameters above can be related to each other as

$$
\mu=\varepsilon^{\alpha},
$$

with $\alpha \geq 0$, meaning that the limiting case $\alpha=0$, associated with a non-contrast setup, is also incorporated.

Using the assumptions above, we establish a two-parametric asymptotic approach in order to investigate the validity of the theory of thin plates. Indeed, it is suggested by physical intuition that in case of a hard thin coating resting on a soft substrate, the model of a thin elastic plate is a reasonable approximation. In this paper, we aim at justification of this model depending on the range of parameter $\alpha$. According to the theory, the deflection of a plate is governed by a bi-harmonic equation

$$
D \Delta^{2} W=P+P_{r}
$$


where $W$ is the deflection of the layer, $\Delta^{2}=\frac{\partial^{4}}{\partial x_{1}^{4}}+2 \frac{\partial^{4}}{\partial x_{1}^{2} \partial x_{2}^{2}}+\frac{\partial^{4}}{\partial x_{2}^{4}}$ is a bi-harmonic operator, $P_{r}$ is reaction of the substrate, $D=\frac{E h^{3}}{12\left(1-\nu^{2}\right)}$ is flexural rigidity, with $E$ and $\nu$ denoting the Young's modulus and Poisson's ratio, respectively.

Let us introduce the dimensionless scaling

$$
\xi_{k}=\frac{x_{k}}{a}, \quad \xi_{3}^{-}=\frac{x_{3}}{h}, \quad 0 \leq x_{3} \leq h, \quad \xi_{3}^{+}=\frac{x_{3}-h}{a}, \quad x_{3} \geq h .
$$

Then, the governing relations for the layer and the half-space are

$$
\begin{aligned}
& \sigma_{i 1,1}^{-}+\sigma_{i 2,2}^{-}+\frac{a}{h} \sigma_{i 3,3}^{-}=0, \\
& \sigma_{k k}^{-}=\frac{\lambda^{-}+2 \mu^{-}}{a} u_{k, k}^{-}+\frac{\lambda^{-}}{a} u_{l, l}^{-}+\frac{\lambda^{-}}{h} u_{3,3}^{-}, \\
& \sigma_{33}^{-}=\frac{\lambda^{-}}{a} u_{1,1}^{-}+\frac{\lambda^{-}}{a} u_{2,2}^{-}+\frac{\lambda^{-}+2 \mu^{-}}{h} u_{3,3}^{-}, \\
& \sigma_{12}^{-}=\frac{\mu^{-}}{a}\left(u_{1,2}^{-}+u_{2,1}^{-}\right), \\
& \sigma_{k 3}^{-}=\frac{\mu^{-}}{h}\left(u_{k, 3}^{-}+\frac{h}{a} u_{3, k}^{-}\right),
\end{aligned}
$$

and

$$
\begin{aligned}
& \sigma_{i 1,1}^{+}+\sigma_{i 2,2}^{+}+\sigma_{i 3,3}^{+}=0, \\
& \sigma_{k k}^{+}=\frac{1}{a}\left(\left(\lambda^{+}+2 \mu^{+}\right) u_{k, k}^{+}+\lambda^{+} u_{l, l}^{+}+\lambda^{+} u_{3,3}^{+}\right), \\
& \sigma_{33}^{+}=\frac{1}{a}\left(\lambda^{+} u_{1,1}^{+}+\lambda^{+} u_{2,2}^{+}+\left(\lambda^{+}+2 \mu^{+}\right) u_{3,3}^{+}\right), \\
& \sigma_{12}^{+}=\frac{\mu^{+}}{a}\left(u_{1,2}^{+}+u_{2,1}^{+}\right), \\
& \sigma_{k 3}^{+}=\frac{\mu^{+}}{a}\left(u_{k, 3}^{+}+u_{3, k}^{+}\right),
\end{aligned}
$$

see e.g. [1], where $u_{i}^{ \pm}$are displacements, $\sigma_{i j}$ are stresses, $\delta_{i j}$ is Kronecker's delta, $\lambda^{ \pm}$are Lamé moduli, and comma indicates differentiation. Here and below, $i, j=1,2,3, l, k=1,2 ; l \neq k$.

The boundary conditions, modeling vertical force at the surface of the layer, and continuity conditions at the interface are

$$
\begin{array}{lrl}
\sigma_{33}^{-}=-P, & \sigma_{k 3}^{-}=0, & \xi_{3}^{-}=0, \\
u_{i}^{-}=u_{i}^{+}, & \sigma_{i 3}^{-}=\sigma_{i 3}^{+}, & \xi_{3}^{-}=1 .
\end{array}
$$

The decay conditions of the displacements $u_{i}^{+} \rightarrow 0$ as $\xi_{3}^{-} \rightarrow \infty$ are also assumed.

\section{Toy plane strain problem}

We start with investigation of a plane strain problem for a vertical harmonic force

$$
P=A \mu^{-} \cos \xi_{1}
$$

where $A$ is constant amplitude, see Figure 2 .

The governing equations follow from (6) and (7), with $u_{2}^{ \pm}=0$ and $\frac{\partial}{\partial \xi_{2}}=0$, i.e.

$$
\begin{aligned}
& \left(\lambda^{-}+2 \mu^{-}\right) \varepsilon^{2} u_{1,11}^{-}+\left(\lambda^{-}+\mu^{-}\right) \varepsilon u_{3,13}^{-}+\mu^{-} u_{1,33}^{-}=0 \\
& \mu^{-} \varepsilon^{2} u_{3,11}^{-}+\left(\lambda^{-}+\mu^{-}\right) \varepsilon u_{1,13}^{-}+\left(\lambda^{-}+2 \mu^{-}\right) u_{3,33}^{-}=0 \\
& \left(\lambda^{+}+2 \mu^{+}\right) u_{1,11}^{+}+\left(\lambda^{+}+\mu^{+}\right) u_{3,13}^{+}+\mu^{+} u_{1,33}^{+}=0 \\
& \mu^{+} u_{3,11}^{+}+\left(\lambda^{+}+\mu^{+}\right) u_{1,13}^{+}+\left(\lambda^{+}+2 \mu^{+}\right) u_{3,33}^{+}=0
\end{aligned}
$$




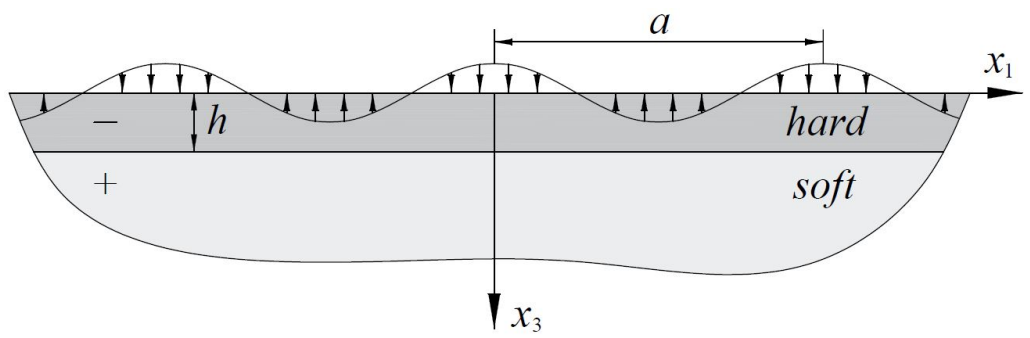

FiguRE 2. Toy plain strain problem.

The boundary and continuity conditions (8) become

$$
\begin{aligned}
& \sigma_{33}^{-}=-A \mu^{-} \cos \xi_{1}, \quad \sigma_{13}^{-}=0, \quad \xi_{3}^{-}=0, \\
& u_{n}^{-}=u_{n}^{+}, \quad \sigma_{13}^{-}=\sigma_{13}^{+}, \quad \xi_{3}^{-}=1,
\end{aligned}
$$

where $n=1,3$, with the decay conditions $u_{n}^{+} \rightarrow 0$ as $x_{3} \rightarrow \infty$.

The solution of the stated problem is given in Appendix 1, see (78) with (80)-(84) for the displacements, whereas the stresses are obtained by substituting the latter into (6) and (7). Using the results together with $(2),(3)$, the leading order asymptotic behaviour of the displacement and stress components is derived, see Table 1.

\begin{tabular}{ccccccccccc}
\hline & $u_{1}^{-}$ & $u_{3}^{-}$ & $\sigma_{13}^{-}$ & $\sigma_{33}^{-}$ & $\sigma_{11}^{-}$ & $u_{1}^{+}$ & $u_{3}^{+}$ & $\sigma_{13}^{+}$ & $\sigma_{33}^{+}$ & $\sigma_{11}^{+}$ \\
\hline$\alpha \geq 3$ & $\varepsilon^{-3}$ & $\varepsilon^{-4}$ & $\varepsilon^{-1}$ & 1 & $\varepsilon^{-2}$ & $\varepsilon^{-4}$ & $\varepsilon^{-4}$ & $\varepsilon^{\alpha-3}$ & $\varepsilon^{\alpha-3}$ & $\varepsilon^{\alpha-3}$ \\
\hline $2 \leq \alpha \leq 3$ & $\varepsilon^{-\alpha}$ & $\varepsilon^{-\alpha-1}$ & $\varepsilon^{2-\alpha}$ & 1 & $\varepsilon^{1-\alpha}$ & $\varepsilon^{-\alpha-1}$ & $\varepsilon^{-\alpha-1}$ & 1 & 1 & 1 \\
\hline $1 \leq \alpha \leq 2$ & $\varepsilon^{-2}$ & $\varepsilon^{-\alpha-1}$ & 1 & 1 & $\varepsilon^{-1}$ & $\varepsilon^{-\alpha-1}$ & $\varepsilon^{-\alpha-1}$ & 1 & 1 & 1 \\
\hline $0 \leq \alpha \leq 1$ & $\varepsilon^{-\alpha-1}$ & $\varepsilon^{-\alpha-1}$ & $\varepsilon^{1-\alpha}$ & 1 & $\varepsilon^{-\alpha}$ & $\varepsilon^{-\alpha-1}$ & $\varepsilon^{-\alpha-1}$ & 1 & 1 & 1 \\
\hline
\end{tabular}

TABLE 1. Asymptotic behaviour of displacement and stress components.

In more detail, considering only the leading order relation between vertical displacement $u_{3}^{-}$and prescribed load (9) at the upper face of the layer $\xi_{3}^{-}=0$, we have

$$
k=\frac{P}{u_{3}^{-}}= \begin{cases}\frac{\mu^{-}\left(\gamma^{-}-1\right) \varepsilon^{4}}{h \gamma^{-}}, & \alpha>3, \\ \frac{\mu^{-}\left(\gamma^{-}-1-\gamma^{+}+7 \gamma^{-} \gamma^{+}\right) \varepsilon^{4}}{3 h \gamma^{-}\left(1+\gamma^{+}\right)}, & \alpha=3, \\ \frac{2 \mu^{-} \gamma^{+} \varepsilon^{\alpha+1}}{h\left(1+\gamma^{+}\right)}, & 1<\alpha<3, \\ \frac{\mu^{-}\left(\gamma^{-}\left(6 \gamma^{+}-2\right)-4 \gamma^{+}\right) \varepsilon^{2}}{h\left(\gamma^{-}\left(2+3 \gamma^{+}\right)-2\left(1+\gamma^{+}\right)\right)}, & \alpha=1, \\ \frac{2 \mu^{-}\left(\gamma^{+}-1\right) \varepsilon^{\alpha+1}}{h \gamma^{+}}, & \alpha<1,\end{cases}
$$

where $\gamma^{ \pm}=\frac{\lambda^{ \pm}+2 \mu^{ \pm}}{\mu^{ \pm}}$

As a result, the case $\alpha>3$ clearly stands out, when the coefficient $k$ is independent of the parameters of substrate. This indicates that the equation of plate bending may be expected at leading order as a relation involving vertical displacement $u_{3}^{-}$and the applied load $P$. 


\section{Asymptotic analysis}

In this section we develop an asymptotic scheme based on the method of direct asymptotic integration of equations of 3D elasticity, see [10,13]. Note that the scaling required for this procedure follows from the orders of displacements and stresses in Table 1.

\subsection{Case $\alpha \geq 3\left(\mu \lesssim \varepsilon^{3}\right)$}

First, we scale the displacements and stresses for the layer using the data in the first line of Table 1. Therefore, we have

$$
\begin{array}{llll}
u_{k}^{-}=h \varepsilon^{-3} u_{k}^{*-}, & \sigma_{k k}^{-}=\mu^{-} \varepsilon^{-2} \sigma_{k k}^{*-}, & \sigma_{12}^{-}=\sigma_{21}^{-}=\mu^{-} \varepsilon^{-2} \sigma_{12}^{*-}, & P=\mu^{-} p^{*}, \\
u_{3}^{-}=h \varepsilon^{-4} u_{3}^{*-}, & \sigma_{33}^{-}=\mu^{-} \sigma_{33}^{*-}, & \sigma_{k 3}^{-}=\sigma_{3 k}^{-}=\mu^{-} \varepsilon^{-1} \sigma_{k 3}^{*-} . &
\end{array}
$$

Here and below, we assume all quantities with the asterisk to be of the same asymptotic order. In view of (13), governing equations (6) become

$$
\begin{aligned}
& \sigma_{i 1,1}^{*-}+\sigma_{i 2,2}^{*-}+\sigma_{i 3,3}^{*-}=0, \\
& \varepsilon^{2} \sigma_{k k}^{*-}=\varepsilon^{2} \gamma^{-} u_{k, k}^{*-}+\varepsilon^{2}\left(\gamma^{-}-2\right) u_{l, l}^{*-}+\left(\gamma^{-}-2\right) u_{3,3}^{*-}, \\
& \varepsilon^{4} \sigma_{33}^{*-}=\varepsilon^{2}\left(\gamma^{-}-2\right) u_{1,1}^{*-}+\varepsilon^{2}\left(\gamma^{-}-2\right) u_{2,2}^{*-}+\gamma^{-} u_{3,3}^{*-}, \\
& \sigma_{12}^{*-}=u_{1,2}^{*-}+u_{2,1}^{*-}, \\
& \varepsilon^{2} \sigma_{k 3}^{*-}=u_{k, 3}^{*-}+u_{3, k}^{*-} .
\end{aligned}
$$

Eliminating the term $u_{3,3}^{*-}$ from $(14)_{2}$ and $(14)_{3}$, we deduce

$$
\sigma_{k k}^{*-} \gamma^{-}-\varepsilon^{2}\left(\gamma^{-}-2\right) \sigma_{33}^{*-}=4\left(\gamma^{-}-1\right) u_{k, k}^{*-}+2\left(\gamma^{-}-2\right) u_{l, l}^{*-} .
$$

Using the scaling for the half-space given by

$$
u_{i}^{+}=h \varepsilon^{-4} u_{i}^{*+}, \quad \sigma_{i j}^{+}=\sigma_{j i}^{+}=\mu^{-} \varepsilon^{\alpha-3} \sigma_{i j}^{*+},
$$

equations (7) may be rewritten as

$$
\begin{aligned}
& \sigma_{i 1,1}^{*+}+\sigma_{i 2,2}^{*+}+\sigma_{i 3,3}^{*+}=0, \\
& \sigma_{k k}^{*+}=\gamma^{+} u_{k, k}^{*+}+\left(\gamma^{+}-2\right)\left(u_{l, l}^{*+}+u_{3,3}^{*+}\right), \\
& \sigma_{33}^{*+}=\gamma^{+} u_{3,3}^{*+}+\left(\gamma^{+}-2\right)\left(u_{1,1}^{*+}+u_{2,2}^{*+}\right), \\
& \sigma_{12}^{*+}=u_{1,2}^{*+}+u_{2,1}^{*+}, \\
& \sigma_{k 3}^{*+}=u_{k, 3}^{*+}+u_{3, k}^{*+} .
\end{aligned}
$$

Boundary and continuity conditions (8) take the form

$$
\sigma_{33}^{*-}=-p^{*}, \quad \sigma_{k 3}^{*-}=0
$$

at $\xi_{3}^{-}=0$, and

$$
\varepsilon u_{k}^{*-}=u_{k}^{*+}, \quad u_{3}^{*-}=u_{3}^{*+}, \quad \sigma_{k 3}^{*-}=\varepsilon^{\alpha-2} \sigma_{k 3}^{*+}, \quad \sigma_{33}^{*-}=\varepsilon^{\alpha-3} \sigma_{33}^{*+} .
$$

at $\xi^{-}=1$.

In (19), $\sigma_{33}^{*-} \gg \sigma_{33}^{*+}$ at $\alpha>3$ whereas $\sigma_{33}^{*-} \sim \sigma_{33}^{*+}$ at $\alpha=3$, therefore we consider these subcases separately.

4.1.1. Subcase $\alpha>3\left(\mu \ll \varepsilon^{3}\right)$. Expand the displacements and stresses of the layer in asymptotic series

$$
\left(\begin{array}{l}
u_{i}^{*-} \\
\sigma_{i j}^{*-}
\end{array}\right)=\left(\begin{array}{l}
u_{i}^{-(0)} \\
\sigma_{i j}^{-(0)}
\end{array}\right)+\ldots
$$


Hence, at leading order, we get from (14)

$$
\begin{aligned}
& \sigma_{i 1,1}^{-(0)}+\sigma_{i 2,2}^{-(0)}+\sigma_{i 3,3}^{-(0)}=0, \\
& u_{3,3}^{-(0)}=0, \\
& \sigma_{12}^{-(0)}=u_{1,2}^{-(0)}+u_{2,1}^{-(0)}, \\
& u_{k, 3}^{-(0)}+u_{3, k}^{-(0)}=0, \\
& \sigma_{k k}^{-(0)}=\kappa u_{k, k}^{-(0)}+(\kappa-2) u_{l, l}^{-(0)},
\end{aligned}
$$

where

$$
\kappa=4\left(1-\frac{1}{\gamma^{-}}\right),
$$

subject to the boundary conditions at $\xi_{3}^{-}=0$

$$
\sigma_{33}^{-(0)}=-p^{*}, \quad \sigma_{k 3}^{-(0)}=0,
$$

and the continuity conditions at $\xi_{3}^{-}=1$

$$
u_{k}^{+(0)}=0, \quad u_{3}^{-(0)}=u_{3}^{+(0)}, \quad \sigma_{i 3}^{-(0)}=0 .
$$

First, $(21)_{2}$ implies

$$
u_{3}^{-(0)}=w,
$$

where $w$ is an arbitrary function of variables $\xi_{1}$ and $\xi_{2}$ representing the deflection of the layer. Then, on integrating $(21)_{4}$, we deduce

$$
u_{k}^{-(0)}=-\xi_{3}^{-} \frac{\partial w}{\partial \xi_{k}}+F_{1},
$$

where $F_{1}=F_{1}\left(\xi_{1}, \xi_{2}\right)$ is an arbitrary function of $\xi_{1}$ and $\xi_{2}$. Substituting the latter into $(21)_{3},(21)_{5}$, using $(21)_{1}$ and satisfying conditions $(23)_{2}$ and $(24)_{3}$, we infer

$$
F_{1}=\frac{1}{2} \frac{\partial w}{\partial \xi_{k}}
$$

Therefore,

$$
u_{k}^{-(0)}=\frac{\partial w}{\partial \xi_{k}}\left(\frac{1}{2}-\xi_{3}^{-}\right)
$$

and

$$
\begin{aligned}
\sigma_{k k}^{-(0)} & =\left(\frac{1}{2}-\xi_{3}^{-}\right)\left(\kappa \frac{\partial^{2} w}{\partial \xi_{k}^{2}}+(\kappa-2) \frac{\partial^{2} w}{\partial \xi_{l}^{2}}\right), \\
\sigma_{12}^{-(0)} & =\left(1-2 \xi_{3}^{-}\right) \frac{\partial^{2} w}{\partial \xi_{1} \partial \xi_{2}}, \\
\sigma_{k 3}^{-(0)} & =\frac{\kappa}{2} \xi_{3}^{-}\left(\xi_{3}^{-}-1\right)\left(\frac{\partial^{3} w}{\partial \xi_{k}^{3}}+\frac{\partial^{3} w}{\partial \xi_{k} \partial \xi_{l}^{2}}\right) .
\end{aligned}
$$

Finally, from $(29)_{3}$ and $(21)_{1}$, and satisfying the continuity condition $(24)_{3}$, we obtain for the vertical stress

$$
\sigma_{33}^{-(0)}=-\frac{\kappa}{12}\left(\left(\xi_{3}^{-}\right)^{2}\left(2 \xi_{3}^{-}-3\right)+1\right) \Delta_{*}^{2} w,
$$

where $\Delta_{*}^{2}=\frac{\partial^{4}}{\partial \xi_{1}^{4}}+2 \frac{\partial^{4}}{\partial \xi_{1}^{2} \partial \xi_{2}^{2}}+\frac{\partial^{4}}{\partial \xi_{2}^{4}}$.

Using the condition $(23)_{1}$ at $\xi_{3}^{-}=0$, we have

$$
\frac{\kappa}{12} \Delta_{*}^{2} w=p^{*}
$$

which in dimensional form coincides with the equation (4) of plate bending with $P_{r}=0$. 
4.1.2. Subcase $\alpha=3\left(\mu \sim \varepsilon^{3}\right)$. Here, we begin with same leading order governing equations for the layer (21) and boundary conditions (23), while continuity conditions at $\xi_{3}^{-}=1$ take the form

$$
u_{k}^{+(0)}=0, \quad u_{3}^{-(0)}=u_{3}^{+(0)}, \quad \sigma_{k 3}^{-(0)}=0, \quad \sigma_{33}^{-(0)}=\sigma_{33}^{+(0)} .
$$

Following a similar procedure, the quantities $u_{3}^{-(0)}, u_{k}^{-(0)}$ are found as $(25),(28)$, respectively, and $\sigma_{k k}^{-(0)}, \sigma_{12}^{-(0)} \sigma_{k 3}^{-(0)}$ as $(29)$. Then, from $(21)_{1}$, we obtain

$$
\sigma_{33}^{-(0)}=-\frac{\kappa}{12}\left(\xi_{3}^{-}\right)^{2}\left(2 \xi_{3}^{-}-3\right) \Delta_{*}^{2} w+F_{2},
$$

where $F_{2}$ is an arbitrary function of $\xi_{1}$ and $\xi_{2}$. The vertical stress at the interface is given by

$$
\left.\sigma_{33}^{-(0)}\right|_{\xi_{3}^{-}=1}=\frac{\kappa}{12} \Delta_{*}^{2} w+F_{2} .
$$

Due to continuity condition $(32)_{4}$ at $\xi_{3}^{-}=1$ we get

$$
F_{2}=\left.\sigma_{33}^{+(0)}\right|_{\xi_{3}^{-}=1}-\frac{\kappa}{12} \Delta_{*}^{2} w .
$$

Therefore,

$$
\sigma_{33}^{-(0)}=-\frac{\kappa}{12}\left(\left(\xi_{3}^{-}\right)^{2}\left(2 \xi_{3}^{-}-3\right)+1\right) \Delta_{*}^{2} w+\left.\sigma_{33}^{+(0)}\right|_{\xi_{3}^{-}=1},
$$

which, on satisfying $(23)_{1}$ at $\xi_{3}^{-}=0$, implies

$$
\frac{\kappa}{12} \Delta_{*}^{2} w=p^{*}+\left.\sigma_{33}^{+(0)}\right|_{\xi_{3}^{-}=1} .
$$

The equation above demonstrates that at $\alpha=3$ the plate bending theory is still valid, but in contrast to the subcase $\alpha>3$, the half-space reaction $P_{r}$ is now a nonzero value.

4.2. Case $2 \leq \alpha<3\left(\varepsilon^{3} \lesssim \mu \ll \varepsilon^{2}\right)$

The scaling for the layer is now given by (see line 2 in Table 1)

$$
\begin{array}{llll}
u_{k}^{-}=h \varepsilon^{-\alpha} u_{k}^{*-}, & \sigma_{k k}^{-}=\mu^{-} \varepsilon^{1-\alpha} \sigma_{k k}^{*-}, & \sigma_{12}^{-}=\sigma_{21}^{-}=\mu^{-} \varepsilon^{1-\alpha} \sigma_{12}^{*-}, & P=\mu^{-} p^{*}, \\
u_{3}^{-}=h \varepsilon^{-\alpha-1} u_{3}^{*-}, & \sigma_{33}^{-}=\mu^{-} \sigma_{33}^{*-}, & \sigma_{k 3}^{-}=\sigma_{3 k}^{-}=\mu^{-} \varepsilon^{2-\alpha} \sigma_{k 3}^{*-} . &
\end{array}
$$

Hence, due to (38), equations (6) become

$$
\begin{aligned}
& \sigma_{k 1,1}^{*-}+\sigma_{k 2,2}^{*-}+\sigma_{k 3,3}^{*-}=0, \\
& \varepsilon^{3-\alpha}\left(\sigma_{13,1}^{*-}+\sigma_{23,2}^{*-}\right)+\sigma_{33,3}^{*-}=0, \\
& \varepsilon^{2} \sigma_{k k}^{*-}=\varepsilon^{2} \gamma^{-} u_{k, k}^{*-}+\varepsilon^{2}\left(\gamma^{-}-2\right) u_{l, l}^{*-}+\left(\gamma^{-}-2\right) u_{3,3}^{*-}, \\
& \varepsilon^{\alpha+1} \sigma_{33}^{*-}=\varepsilon^{2}\left(\gamma^{-}-2\right) u_{1,1}^{*-}+\varepsilon^{2}\left(\gamma^{-}-2\right) u_{2,2}^{*-}+\gamma^{-} u_{3,3}^{*-}, \\
& \sigma_{12}^{*-}=u_{1,2}^{*-}+u_{2,1}^{*-}, \\
& \varepsilon^{2} \sigma_{k 3}^{*-}=u_{k, 3}^{*-}+u_{3, k}^{*-} .
\end{aligned}
$$

Similarly to the previous Subsection 4.1, we deduce from $(39)_{3}$ and $(39)_{4}$

$$
\sigma_{k k}^{*-} \gamma^{-}-\varepsilon^{\alpha-1}\left(\gamma^{-}-2\right) \sigma_{33}^{*-}=4\left(\gamma^{-}-1\right) u_{k, k}^{*-}+2\left(\gamma^{-}-2\right) u_{l, l}^{*-} .
$$

The scaling for the half-space here and below takes the form

$$
u_{i}^{+}=h \varepsilon^{-\alpha-1} u_{i}^{*+}, \quad \sigma_{i j}^{+}=\sigma_{j i}^{+}=\mu^{-} \sigma_{i j}^{*+},
$$

leading to equations (17).

Boundary conditions are once again represented as (18) and the continuity conditions are

$$
\varepsilon u_{k}^{*-}=u_{k}^{*+}, \quad u_{3}^{*-}=u_{3}^{*+}, \quad \sigma_{k 3}^{*-}=\varepsilon^{\alpha-2} \sigma_{k 3}^{*+}, \quad \sigma_{33}^{*-}=\sigma_{33}^{*+} .
$$

Below, we deal with the subcases $2<\alpha<3$ and $\alpha=2$ separately. 
4.2.1. Subcase $2<\alpha<3\left(\varepsilon^{3} \ll \mu \ll \varepsilon^{2}\right)$. The leading order governing equations are

$$
\begin{aligned}
& \sigma_{k 1,1}^{-(0)}+\sigma_{k 2,2}^{-(0)}+\sigma_{k 3,3}^{-(0)}=0, \\
& \sigma_{33,3}^{-(0)}=0, \\
& u_{3,3}^{-(0)}=0, \\
& \sigma_{12}^{-(0)}=u_{1,2}^{-(0)}+u_{2,1}^{-(0)}, \\
& u_{k, 3}^{-(0)}+u_{3, k}^{-(0)}=0, \\
& \sigma_{k k}^{-(0)}=\kappa u_{k, k}^{-(0)}+(\kappa-2) u_{l, l}^{-(0)},
\end{aligned}
$$

subject to boundary conditions (23), and the following continuity conditions at $\xi_{3}^{-}=1$

$$
u_{k}^{+(0)}=0, \quad u_{3}^{-(0)}=u_{3}^{+(0)}, \quad \sigma_{k 3}^{-(0)}=0, \quad \sigma_{33}^{-(0)}=\sigma_{33}^{+(0)} .
$$

As in Subsection 4.1, the quantities $u_{3}^{-(0)}, u_{k}^{-(0)}$ are found in the form (25), (28), respectively, and $\sigma_{k k}^{-(0)}, \sigma_{12}^{-(0)} \sigma_{k 3}^{-(0)}$ are obtained as (29). Then, integrating $(43)_{2}$ with respect to $\xi_{3}^{-}$and satisfying $(23)_{1}$, we obtain

$$
\sigma_{33}^{-(0)}=-p^{*}
$$

It is clear from (45) that in this case the applied load $p^{*}$ is transmitted to the interface and is no longer connected with the layer deflection directly. Thus, the value of the deflection and, subsequently, the rest of the stresses and displacements, strongly depend on the value of the interfacial displacement $u_{3}^{+(0)}$, due to $(44)_{2}$, which indicates violation of the plate bending theory.

4.2.2. Subcase $\alpha=2\left(\mu \sim \varepsilon^{2}\right)$. The leading order equations are again given by (43) with boundary conditions (23), and the continuity conditions at $\xi_{3}^{-}=1$ are written as

$$
u_{k}^{+(0)}=0, \quad u_{3}^{-(0)}=u_{3}^{+(0)}, \quad \sigma_{i 3}^{-(0)}=\sigma_{i 3}^{+(0)},
$$

leading to vertical displacement $u_{3}^{-(0)}$ and stress $\sigma_{33}^{-(0)}$ expressed as (25) and (45), respectively. Using $(43)_{5}$, we deduce

$$
\begin{aligned}
& u_{1}^{-(0)}=-\xi_{3}^{-} \frac{\partial w}{\partial \xi_{1}}+F_{3}, \\
& u_{2}^{-(0)}=-\xi_{3}^{-} \frac{\partial w}{\partial \xi_{2}}+F_{4},
\end{aligned}
$$

where $F_{3}$ and $F_{4}$ are arbitrary functions of $\xi_{1}$ and $\xi_{2}$. Therefore, $(43)_{6}$ implies

$$
\begin{aligned}
\sigma_{11}^{-(0)} & =-\kappa \xi_{3}^{-} \frac{\partial^{2} w}{\partial \xi_{1}^{2}}+\kappa \frac{\partial F_{3}}{\partial \xi_{1}}-(\kappa-2) \xi_{3}^{-} \frac{\partial^{2} w}{\partial \xi_{2}^{2}}+(\kappa-2) \frac{\partial F_{4}}{\partial \xi_{2}}, \\
\sigma_{22}^{-(0)} & =-(\kappa-2) \xi_{3}^{-} \frac{\partial^{2} w}{\partial \xi_{1}^{2}}+(\kappa-2) \frac{\partial F_{3}}{\partial \xi_{1}}-\kappa \xi_{3}^{-} \frac{\partial^{2} w}{\partial \xi_{2}^{2}}+\kappa \frac{\partial F_{4}}{\partial \xi_{2}}
\end{aligned}
$$

and $(43)_{4}$ yields

$$
\sigma_{12}^{-(0)}=-2 \xi_{3}^{-} \frac{\partial^{2} w}{\partial \xi_{1} \xi_{2}}+\frac{\partial F_{3}}{\partial \xi_{2}}+\frac{\partial F_{4}}{\partial \xi_{1}} .
$$

From $(43)_{1}$, taking into account (48) and (49), and satisfying $(23)_{2}$, we arrive at

$$
\begin{aligned}
& \sigma_{13}^{-(0)}=\frac{\kappa}{2}\left(\xi_{3}^{-}\right)^{2} \frac{\partial^{3} w}{\partial \xi_{1}^{3}}-\kappa \xi_{3}^{-} \frac{\partial^{2} F_{3}}{\partial \xi_{1}^{2}}+\frac{\kappa}{2}\left(\xi_{3}^{-}\right)^{2} \frac{\partial^{3} w}{\partial \xi_{1} \partial \xi_{2}^{2}}-(\kappa-1) \xi_{3}^{-} \frac{\partial^{2} F_{4}}{\partial \xi_{1} \partial \xi_{2}}-\xi_{3}^{-} \frac{\partial^{2} F_{3}}{\partial \xi_{2}^{2}}, \\
& \sigma_{23}^{-(0)}=\frac{\kappa}{2}\left(\xi_{3}^{-}\right)^{2} \frac{\partial^{3} w}{\partial \xi_{1}^{2} \partial \xi_{2}}-(\kappa-1) \xi_{3}^{-} \frac{\partial^{2} F_{3}}{\partial \xi_{1} \partial \xi_{2}}+\frac{\kappa}{2}\left(\xi_{3}^{-}\right)^{2} \frac{\partial^{3} w}{\partial \xi_{2}^{3}}-\kappa \xi_{3}^{-} \frac{\partial^{2} F_{4}}{\partial \xi_{2}^{2}}-\xi_{3}^{-} \frac{\partial^{2} F_{4}}{\partial \xi_{1}^{2}},
\end{aligned}
$$

where functions $F_{3}$ and $F_{4}$ can be found from solving the problem for the half-space and using continuity conditions $(46)_{3}$. 
4.3. Case $1 \leq \alpha<2\left(\varepsilon^{2} \lesssim \mu \ll \varepsilon\right)$

The scaling for the layer is taken as

$$
\begin{array}{lll}
u_{3}^{-}=h \varepsilon^{-\alpha-1} u_{3}^{*-}, & u_{k}^{-}=h \varepsilon^{-2} u_{k}^{*-}, & \sigma_{12}^{-}=\sigma_{21}^{-}=\mu^{-} \varepsilon^{-1} \sigma_{12}^{*-}, \\
P=\mu^{-} p^{*}, & \sigma_{k k}^{-}=\mu^{-} \varepsilon^{-1} \sigma_{k k}^{*-}, & \sigma_{i 3}^{-}=\sigma_{3 i}^{-}=\mu^{-} \sigma_{k 3}^{*-} .
\end{array}
$$

Substituting the latter into governing equations (6), we have

$$
\begin{aligned}
& \sigma_{k 1,1}^{*-}+\sigma_{k 2,2}^{*-}+\sigma_{k 3,3}^{*-}=0, \\
& \varepsilon\left(\sigma_{13,1}^{*-}+\sigma_{23,2}^{*-}\right)+\sigma_{33,3}^{*-}=0, \\
& \varepsilon^{\alpha} \sigma_{k k}^{*-}=\varepsilon^{\alpha} \gamma^{-} u_{k, k}^{*-}+\varepsilon^{\alpha}\left(\gamma^{-}-2\right) u_{l, l}^{*-}+\left(\gamma^{-}-2\right) u_{3,3}^{*-}, \\
& \varepsilon^{\alpha+1} \sigma_{33}^{*-}=\varepsilon^{\alpha}\left(\gamma^{-}-2\right) u_{1,1}^{*-}+\varepsilon^{\alpha}\left(\gamma^{-}-2\right) u_{2,2}^{*-}+\gamma^{-} u_{3,3}^{*-}, \\
& \sigma_{12}^{*-}=u_{1,2}^{*-}+u_{2,1}^{*-}, \\
& \varepsilon^{2} \sigma_{k 3}^{*-}=u_{k, 3}^{*-}+\varepsilon^{2-\alpha} u_{3, k}^{*-},
\end{aligned}
$$

with the following additional equation obtained from $(52)_{3}$ and $(52)_{4}$

$$
\sigma_{k k}^{*-} \gamma^{-}-\varepsilon\left(\gamma^{-}-2\right) \sigma_{33}^{*-}=4\left(\gamma^{-}-1\right) u_{k, k}^{*-}+2\left(\gamma^{-}-2\right) u_{l, l}^{*-},
$$

this being similar to (15) and (40).

The boundary conditions are taken as (18), whereas the continuity conditions at $\xi_{3}^{-}=1$ are

$$
\varepsilon^{\alpha-1} u_{k}^{*-}=u_{k}^{*+}, \quad u_{3}^{*-}=u_{3}^{*+}, \quad \sigma_{i 3}^{*-}=\sigma_{i 3}^{*+} .
$$

We again treat subcases $1<\alpha<2$ and $\alpha=1$ separately.

4.3.1. Subcase $1<\alpha<2\left(\varepsilon^{2} \ll \mu \ll \varepsilon\right)$. Leading order equations are

$$
\begin{aligned}
& \sigma_{k 1,1}^{-(0)}+\sigma_{k 2,2}^{-(0)}+\sigma_{k 3,3}^{-(0)}=0, \\
& \sigma_{33,3}^{-(0)}=0, \\
& u_{3,3}^{-(0)}=0, \\
& \sigma_{12}^{-(0)}=u_{1,2}^{-(0)}+u_{2,1}^{-(0)}, \\
& u_{k, 3}^{-(0)}=0, \\
& \sigma_{k k}^{-(0)}=\kappa u_{k, k}^{-(0)}+(\kappa-2) u_{l, l}^{-(0)},
\end{aligned}
$$

subject to boundary conditions $(23)$ and the continuity conditions at $\xi_{3}^{-}=1$

$$
u_{k}^{+(0)}=0, \quad u_{3}^{-(0)}=u_{3}^{+(0)}, \quad \sigma_{i 3}^{-(0)}=\sigma_{i 3}^{+(0)} .
$$

As before, $u_{3}^{-(0)}$ and $\sigma_{33}^{-(0)}$ are expressed as (25) and (45). It follows from $(55)_{5}$ and $(61)_{1}$ that

$$
\begin{aligned}
& u_{1}^{-(0)}=F_{5}, \\
& u_{2}^{-(0)}=F_{6},
\end{aligned}
$$

resulting in

and

$$
\begin{aligned}
& \sigma_{11}^{-(0)}=\kappa \frac{\partial F_{5}}{\partial \xi_{1}}+(\kappa-2) \frac{\partial F_{6}}{\partial \xi_{2}} \\
& \sigma_{22}^{-(0)}=\kappa \frac{\partial F_{6}}{\partial \xi_{2}}+(\kappa-2) \frac{\partial F_{5}}{\partial \xi_{1}}
\end{aligned}
$$

$$
\sigma_{12}^{-(0)}=\frac{\partial F_{5}}{\partial \xi_{2}}+\frac{\partial F_{6}}{\partial \xi_{1}}
$$

where $F_{5}$ and $F_{6}$ are arbitrary functions of $\xi_{1}$ and $\xi_{2}$. Finally, from $(55)_{1}$, satisfying $(23)_{2}$, we infer

$$
\begin{aligned}
& \sigma_{13}^{-(0)}=-\xi_{3}^{-}\left(\kappa \frac{\partial^{2} F_{5}}{\partial \xi_{1}^{2}}+(\kappa-1) \frac{\partial^{2} F_{6}}{\partial \xi_{1} \partial \xi_{2}}+\frac{\partial^{2} F_{5}}{\partial \xi_{2}^{2}}\right), \\
& \sigma_{23}^{-(0)}=-\xi_{3}^{-}\left(\kappa \frac{\partial^{2} F_{6}}{\partial \xi_{2}^{2}}+(\kappa-1) \frac{\partial^{2} F_{5}}{\partial \xi_{1} \partial \xi_{2}}+\frac{\partial^{2} F_{6}}{\partial \xi_{1}^{2}}\right) .
\end{aligned}
$$

Here, functions $F_{5}$ and $F_{6}$ can again be derived from the continuity conditions $(56)_{3}$. 
4.3.2. Subcase $\alpha=1(\mu \sim \varepsilon)$. Now the leading order governing equations and the boundary conditions are taken as (55) and (23), respectively, and the continuity conditions at $\xi_{3}^{-}=1$ are

$$
u_{i}^{-(0)}=u_{i}^{+(0)}, \quad \sigma_{i 3}^{-(0)}=\sigma_{i 3}^{+(0)} .
$$

As above, $u_{3}^{-(0)}$ and $\sigma_{33}^{-(0)}$ are given by (25) and (45), respectively, whereas $(55)_{5}$ and $(61)_{1}$ imply

$$
u_{k}^{-(0)}=\left.u_{k}^{+(0)}\right|_{\xi_{3}^{-}=1} .
$$

Hence,

$$
\begin{aligned}
& \sigma_{k k}^{-(0)}=\left.\left(\kappa \frac{\partial u_{k}^{+(0)}}{\partial \xi_{k}}+(\kappa-2) \frac{\partial u_{l}^{+(0)}}{\partial \xi_{l}}\right)\right|_{\xi_{3}^{-}=1}, \\
& \sigma_{12}^{-(0)}=\left.\left(\frac{\partial u_{1}^{+(0)}}{\partial \xi_{2}}+\frac{\partial u_{2}^{+(0)}}{\partial \xi_{1}}\right)\right|_{\xi_{3}^{-}=1} .
\end{aligned}
$$

Thus, from $(55)_{1}$, and satisfying $(23)_{2}$, we infer

$$
\sigma_{k 3}^{-(0)}=-\left.\xi_{3}^{-}\left(\kappa \frac{\partial^{2} u_{k}^{+(0)}}{\partial \xi_{k}^{2}}+(\kappa-1) \frac{\partial^{2} u_{l}^{+(0)}}{\partial \xi_{1} \partial \xi_{2}}+\frac{\partial^{2} u_{k}^{+(0)}}{\partial \xi_{l}^{2}}\right)\right|_{\xi_{3}^{-}=1} .
$$

4.4. Case $0 \leq \alpha<1(\varepsilon \lesssim \mu \ll 1)$

The scaling for the layer is

$$
\begin{array}{lll}
u_{i}^{-}=h \varepsilon^{-\alpha-1} u_{i}^{*-}, & \sigma_{33}^{-}=\mu^{-} \sigma_{33}^{*-}, & \sigma_{12}^{-}=\sigma_{21}^{-}=\mu^{-} \varepsilon^{-\alpha} \sigma_{12}^{*-} \\
\sigma_{k k}^{-}=\mu^{-} \varepsilon^{-\alpha} \sigma_{k k}^{*-}, & P=\mu^{-} p^{*}, & \sigma_{k 3}^{-}=\sigma_{3 k}^{-}=\mu^{-} \varepsilon^{1-\alpha} \sigma_{k 3}^{*-}
\end{array}
$$

As a result, the governing equations (6) become

$$
\begin{aligned}
& \sigma_{k 1,1}^{*-}+\sigma_{k 2,2}^{*-}+\sigma_{k 3,3}^{*-}=0, \\
& \varepsilon^{2-\alpha}\left(\sigma_{13,1}^{*-}+\sigma_{23,2}^{*-}\right)+\sigma_{33,3}^{*-}=0, \\
& \varepsilon \sigma_{k k}^{*-}=\varepsilon \gamma^{-} u_{k, k}^{*-}+\varepsilon\left(\gamma^{-}-2\right) u_{l, l}^{*-}+\left(\gamma^{-}-2\right) u_{3,3}^{*-}, \\
& \varepsilon^{\alpha+1} \sigma_{33}^{*-}=\varepsilon\left(\gamma^{-}-2\right) u_{1,1}^{*-}+\varepsilon\left(\gamma^{-}-2\right) u_{2,2}^{*-}+\gamma^{-} u_{3,3}^{*-}, \\
& \sigma_{12}^{*-}=u_{1,2}^{*-}+u_{2,1}^{*-}, \\
& \varepsilon^{2} \sigma_{k 3}^{*-}=u_{k, 3}^{*-}+\varepsilon u_{3, k}^{*-},
\end{aligned}
$$

together with the equation

$$
\sigma_{k k}^{*-} \gamma^{-}-\varepsilon^{\alpha}\left(\gamma^{-}-2\right) \sigma_{33}^{*-}=4\left(\gamma^{-}-1\right) u_{k, k}^{*-}+2\left(\gamma^{-}-2\right) u_{l, l}^{*-},
$$

subject to boundary conditions (18) and the continuity conditions at $\xi_{3}^{-}=1$

$$
u_{i}^{*-}=u_{i}^{*+}, \quad \sigma_{k 3}^{*-}=\varepsilon^{\alpha-1} \sigma_{k 3}^{*+}, \quad \sigma_{33}^{*-}=\sigma_{33}^{*+} .
$$

At leading order the governing equations are

$$
\begin{aligned}
& \sigma_{k 1,1}^{-(0)}+\sigma_{k 2,2}^{-(0)}+\sigma_{k 3,3}^{-(0)}=0, \\
& \sigma_{33,3}^{-(0)}=0, \\
& u_{3,3}^{-(0)}=0, \\
& \sigma_{12}^{-(0)}=u_{1,2}^{-(0)}+u_{2,1}^{-(0)}, \\
& u_{k, 3}^{-(0)}=0, \\
& \sigma_{k k}^{-(0)}-\varepsilon^{\alpha} \frac{\kappa-2}{2} \sigma_{33}^{-(0)}=\kappa u_{k, k}^{-(0)}+(\kappa-2) u_{l, l}^{-(0)},
\end{aligned}
$$

where term $\sigma_{33}^{-(0)}$ can be neglected at $0<\alpha<1$, with boundary conditions (23) and the continuity conditions at $\xi_{3}^{-}=1$

$$
u_{i}^{-(0)}=u_{i}^{+(0)}, \quad \sigma_{k 3}^{+(0)}=0, \quad \sigma_{33}^{-(0)}=\sigma_{33}^{+(0)} .
$$


The stated problem leads to the results for the displacements and vertical stress given by (25), (62) and (45).

\subsection{Approximate formulations for a half-space}

The consideration above demonstrates that the plate bending theory is applicable only for a rather high contrast setup $(\alpha \geq 3)$. At the same time, at $\alpha \leq 3$, it is not possible to treat the layer and the half-space separately. We can, however, formulate boundary conditions at the surface of the half-space instead of solving the full original problem for a layered solid.

At $\alpha=3$, using continuity conditions (32) along with equation (37), the sought for effective boundary conditions at $\xi_{3}^{+}=0$ for the half-space take the form

$$
u_{k}^{+(0)}=0, \quad \sigma_{33}^{+(0)}=\left.\frac{\kappa}{12} \Delta_{*}^{2} u_{3}^{+(0)}\right|_{\xi_{3}^{+}=0}-p^{*} .
$$

Thus, the normal stress is expressed through the vertical displacement at the surface; in doing so, the operator in the right hand side of (71) corresponds to the Kirchhoff plate theory.

At $0 \leq \alpha<3$, the vertical force applied at the upper face of the layer can be transmitted to the interface, see (45). Therefore, at $1<\alpha<3$, using continuity conditions (44), (46) and (56), we arrive at the following mixed boundary conditions along the surface of the half-space $\xi_{3}^{+}=0$

$$
u_{k}^{+(0)}=0, \quad \sigma_{33}^{+(0)}=-p^{*} .
$$

At $\alpha=1$, taking into account expression (64) for shear stresses and continuity conditions (61), the effective boundary conditions at $\xi_{3}^{+}=0$ become

$$
\sigma_{33}^{+(0)}=-p^{*}, \quad \sigma_{k 3}^{+(0)}=-\left.\left(\kappa \frac{\partial^{2} u_{k}^{+(0)}}{\partial \xi_{k}^{2}}+(\kappa-1) \frac{\partial^{2} u_{l}^{+(0)}}{\partial \xi_{1} \partial \xi_{2}}+\frac{\partial^{2} u_{k}^{+(0)}}{\partial \xi_{l}^{2}}\right)\right|_{\xi_{3}^{+}=0} .
$$

Here, the shear stresses are expressed through the horisontal displacements at the surface. These conditions were previously studied in [10] and [28] for a non-contrast case.

Finally, at $0 \leq \alpha<1$, due to continuity conditions (70), we get at $\xi_{3}^{+}=0$

$$
\sigma_{33}^{+(0)}=-p^{*}, \quad \sigma_{k 3}^{+(0)}=0 .
$$

Solutions of plane strain problems for a half-space subject to the boundary conditions above are presented in Appendix 2 with a harmonic load $P=A \mu^{-} \cos \xi_{1}$.

\section{Validation of asymptotic results}

In this section we justify the derived asymptotic results by numerical comparison with the exact solution for a harmonic load.

We reiterate that analysis of the relation between the applied force $p^{*}$ and the deflection of the layer $w$ indicates that the classical equation of plate bending arises only at a relatively high contrast $\left(\alpha \geq 3\right.$ or $\left.\mu \lesssim \varepsilon^{3}\right)$, see (31) and (37).

In case of $\alpha<3$, the plate theory is not valid. Nevertheless, transverse displacement $u_{3}^{-(0)}$ is always uniform at leading order across the thickness of the layer, i.e. $u_{3}^{-(0)}=w$, see (25). Thus, due to continuity conditions, we have

$$
w=\left.u_{3}^{+(0)}\right|_{\xi_{3}^{+}=0}
$$

In this case, the effective boundary conditions for a homogeneous half-space, modeling the presence of the layer, are discussed in Subsection 4.5 for different values of parameter $\alpha$. As a result, we obtain from (75) the deflection of the layer.

Let us now compare asymptotic results with the exact solution of the plane problem for harmonic load $P=A \mu^{-} \cos \xi_{1}$ applied at the surface of a layered half-space $x_{3}=0$. Using relation (31) for $\alpha>3$ and the solutions of the plane problems in Appendix 2 for $0 \leq \alpha \leq 3$, the expression for the coefficient $k$ coincides with leading order exact solution (12). 


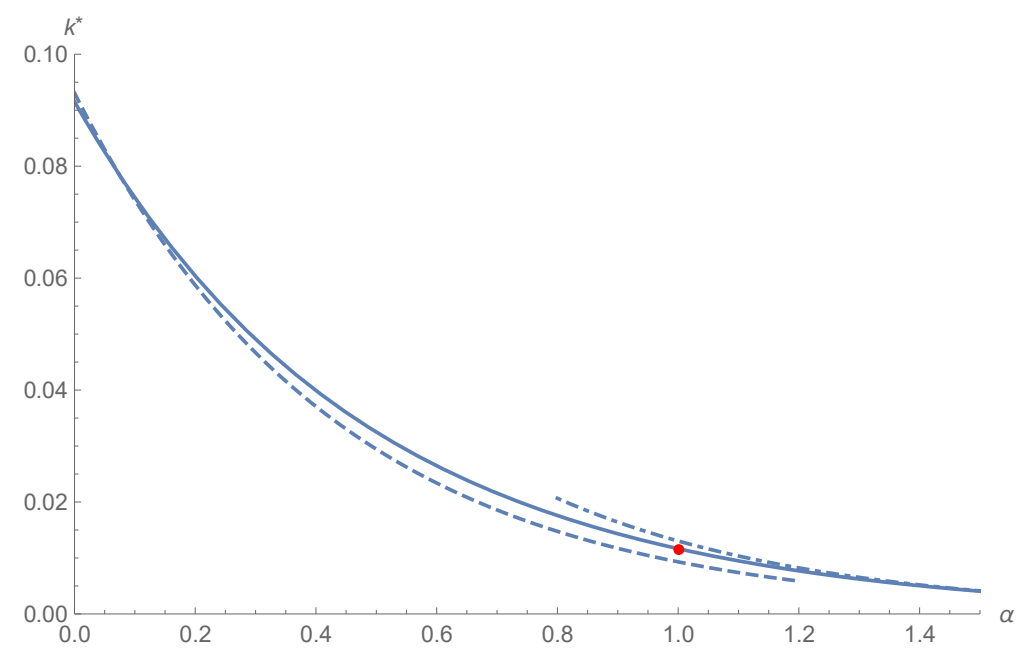

Figure 3. Approximate and exact solutions for harmonic load for $0 \leq \alpha \leq 1.5$.

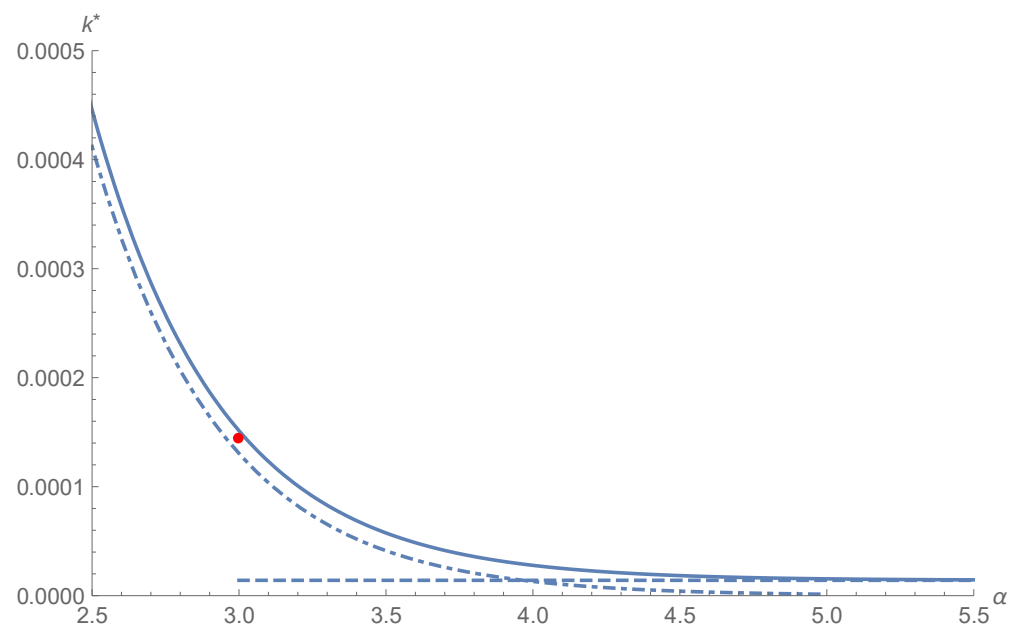

FIgURE 4. Approximate and exact solutions for harmonic load for $2.5 \leq \alpha \leq 5.5$.

As an illustration, in Figures 3 and 4 we present numerical results, where $\alpha=\log _{\varepsilon} \mu$ and also $\nu^{-}=0.25, \nu^{+}=0.3$, and $\varepsilon=h / a=0.1$, for dimensionless coefficient $k^{*}$ expressed as

$$
k^{*}=\frac{h}{\mu^{-}} k
$$

and

$$
k^{*}=\frac{A h}{c_{1}+c_{2}}
$$

for the asymptotic and exact solution, respectively, where constants $c_{1}$ and $c_{2}$ are given by (81), see Appendix 1.

The exact solution is plotted with solid line, while the graphs corresponding to the developed asymptotic approximations are displayed by dashed and dot-dashed lines. The asymptotic results for limiting cases $\alpha=1$ and $\alpha=3$ are displayed by red dots in Figures 3 and 4, respectively. The region $1.5<\alpha<2.5$ is not shown in the Figures since the difference between the associated asymptotic approximation and the exact solution is virtually indistinguishable. 


\section{Conclusion}

In addition to [24] dealing with a soft thin layer covering a half-space, a full two-parametric analysis of the related 3D problem in linear elasticity for a stiff coating is developed. It is confirmed that the Kirchhoff plate theory is only valid for a sufficiently high contrast in the layer and substrate stiffnesses $\left(\alpha \geq 3\right.$ or $\mu \lesssim \varepsilon^{3}$ ). Nevertheless, several approximate formulations for a homogeneous half-space are derived at $\alpha<3$, see boundary conditions (71)-(74). The obtained asymptotic results are compared numerically with the exact solution for a vertical sinusoidal load applied to the upper face of the layer, see Figures 3 and 4.

The considerations in the paper are of interest for a range of problems for coated bodies including modeling of contact interaction. The proposed methodology may be easily extended to anisotropic solids, shell-like coatings, as well as to the case of more sophisticated interfacial conditions. At the same time, incorporating dynamic and nonlinear phenomena in the proposed two-parametric scheme seems to be less straightforward, e.g. see aforementioned papers [6, 14] and also [21, 22, 23] dealing with high-frequency thickness vibration.

\section{Appendix 1}

The displacements for a stated plane strain problem in Section 3 may be found as

$$
u_{1}^{ \pm}=f_{1}^{ \pm}\left(\xi_{3}^{ \pm}\right) \sin \xi_{1}, \quad u_{3}^{ \pm}=f_{3}^{ \pm}\left(\xi_{3}^{ \pm}\right) \cos \xi_{1} .
$$

Then, substituting (78) into (10), we get

$$
\begin{aligned}
& \mu^{-} f_{1}^{-\prime \prime}\left(\xi_{3}^{-}\right)-\left(\lambda^{-}+2 \mu^{-}\right) \varepsilon^{2} f_{1}^{-}\left(\xi_{3}^{-}\right)-\left(\lambda^{-}+\mu^{-}\right) \varepsilon f_{3}^{-^{\prime}}\left(\xi_{3}^{-}\right)=0, \\
& \left(\lambda^{-}+2 \mu^{-}\right) f_{3}^{-\prime \prime}\left(\xi_{3}^{-}\right)+\left(\lambda^{-}+\mu^{-}\right) \varepsilon f_{1}^{-^{\prime}}\left(\xi_{3}^{-}\right)-\mu^{-} \varepsilon^{2} f_{3}^{-}\left(\xi_{3}^{-}\right)=0, \\
& \mu^{+}{f_{1}^{+}}^{+\prime}\left(\xi_{3}^{+}\right)-\left(\lambda^{+}+2 \mu^{+}\right) f_{1}^{+}\left(\xi_{3}^{+}\right)-\left(\lambda^{+}+\mu^{+}\right){f_{3}^{+}}^{\prime}\left(\xi_{3}^{+}\right)=0, \\
& \left(\lambda^{+}+2 \mu^{+}\right) f_{3}^{+\prime \prime}\left(\xi_{3}^{+}\right)+\left(\lambda^{+}+\mu^{+}\right){f_{1}^{+}}^{+}\left(\xi_{3}^{+}\right)-\mu^{+} f_{3}^{+}\left(\xi_{3}^{+}\right)=0 .
\end{aligned}
$$

Therefore, using the latter together with boundary and continuity conditions (11), the solution decaying at infinity is given by

$$
\begin{aligned}
& f_{1}^{-}\left(\xi_{3}^{-}\right)=e^{-\varepsilon \xi_{3}^{-}}\left[c_{2}+c_{4}\left(\xi_{3}^{-}-c_{0}^{-}\right)\right]-e^{\varepsilon \xi_{3}^{-}}\left[c_{1}+c_{3}\left(\xi_{3}^{-}+c_{0}^{-}\right)\right], \\
& f_{3}^{-}\left(\xi_{3}^{-}\right)=\left(c_{1}+c_{3} \xi_{3}^{-}\right) e^{\varepsilon \xi_{3}^{-}}+\left(c_{2}+c_{4} \xi_{3}^{-}\right) e^{-\varepsilon \xi_{3}^{-}} \\
& f_{1}^{+}\left(\xi_{3}^{+}\right)=e^{-\xi_{3}^{+}}\left[c_{5}+c_{6}\left(\xi_{3}^{+}-\varepsilon c_{0}^{+}\right)\right] \\
& f_{3}^{+}\left(\xi_{3}^{+}\right)=\left(c_{5}+c_{6} \xi_{3}^{+}\right) e^{-\xi_{3}^{+}} .
\end{aligned}
$$

The values of $c_{0}^{ \pm}$and $c_{q}, q=1, \ldots, 6$ are

$$
c_{0}^{ \pm}=\frac{1}{\varepsilon}\left(1+\frac{2 \mu^{ \pm}}{\lambda^{ \pm}+\mu^{ \pm}}\right), \quad c_{q}=\frac{A h N_{q}}{D}, \quad q=1, \ldots, 6,
$$

where

$$
\begin{aligned}
N_{1}= & 2 \mathrm{e}^{2 \varepsilon} \varepsilon^{2} B_{7} B_{12}+\left(\gamma^{-}\right)^{2}\left[B_{2}^{+} B_{13}^{-}\left(\mu^{-}\right)^{2}+2\left(\gamma^{+}-2 \mathrm{e}^{2 \varepsilon} \varepsilon B_{3}^{+}\right) \mu^{-} \mu^{+}-B_{1}^{+} B_{13}^{+}\left(\mu^{+}\right)^{2}\right] \\
& +\gamma^{-}\left[B_{1}^{+} B_{14}^{-}\left(\mu^{+}\right)^{2}+2\left(2 \mathrm{e}^{2 \varepsilon} \varepsilon B_{4}^{+}-1\right)-B_{2}^{+} B_{14}^{+}\left(\mu^{-}\right)^{2}\right], \\
N_{2}= & -\mathrm{e}^{2 \varepsilon}\left(2 \varepsilon^{2} B_{7} B_{12}+\gamma^{-}\left[B_{2}^{+} B_{10}^{-}\left(\mu^{-}\right)^{2}-2\left(\mathrm{e}^{2 \varepsilon}+2 B_{4}^{-} \varepsilon\right) \mu^{-} \mu^{+}-B_{1}^{+} B_{10}^{+}\left(\mu^{+}\right)^{2}\right]-\left(\gamma^{-}\right)^{2}\right. \\
& {\left.\left[B_{2}^{+}\left(B_{9}^{-}-2 \varepsilon^{2}\right)\left(\mu^{-}\right)^{2}+2\left(\mathrm{e}^{2 \varepsilon} \gamma^{+}-2 B_{3}^{-} \varepsilon\right) \mu^{-} \mu^{+}+B_{1}^{+}\left(B_{4}^{-}+\mathrm{e}^{2 \varepsilon}+2 \varepsilon^{2}\right)\left(\mu^{+}\right)^{2}\right]\right), } \\
N_{3}= & -\varepsilon B_{1}^{-} B_{7}\left[B_{1}^{-} B_{2}^{+}\left(\mathrm{e}^{2 \varepsilon} B_{4}^{+}-1\right) \mu^{-}+B_{1}^{+}\left(B_{2}^{-}+\mathrm{e}^{2 \varepsilon} B_{1}^{-} B_{4}^{+}\right) \mu^{+}\right], \\
N_{4}= & \varepsilon \mathrm{e}^{2 \varepsilon} B_{1}^{-} B_{12}\left[B_{1}^{-} B_{9}^{-} \mu^{-}+\left(B_{1}^{-} B_{4}^{-}+\mathrm{e}^{2 \varepsilon} B_{2}^{-}\right) \mu^{+}\right], \\
N_{5}= & 2 \mathrm{e}^{\varepsilon} \gamma^{-} \mu^{-}\left[B_{1}^{-} B_{2}^{+}\left(\mathrm{e}^{2 \varepsilon} B_{3}^{+}-B_{3}^{-}\right) \mu^{-}+\left(\mathrm{e}^{2 \varepsilon} B_{11}^{+}-B_{11}^{-}\right) \mu^{+}\right], \\
N_{6}= & 2 \mathrm{e}^{\varepsilon} \gamma^{-} B_{1}^{+} \mu^{-}\left[B_{1}^{-} B_{9}^{-} \mu^{-}+\left(B_{1}^{-} B_{4}^{-}+\mathrm{e}^{2 \varepsilon} B_{2}^{-}\right) \mu^{+}\right],
\end{aligned}
$$

and

$$
\begin{aligned}
D= & 2 \varepsilon\left[\left(B_{1}^{-}\right)^{2} B_{2}^{+}\left(B_{6}-2 \mathrm{e}^{2 \varepsilon} B_{5}\right)\left(\mu^{-}\right)^{2}+2 B_{1}^{-}\left[B_{8}^{-}+\mathrm{e}^{4 \varepsilon} B_{8}^{+}+\mathrm{e}^{2 \varepsilon}\left(4 B_{1}^{-} \varepsilon^{2}-2\right)\right] \mu^{-} \mu^{+}\right. \\
& \left.+B_{1}^{+}\left[B_{1}^{-} B_{2}^{-} B_{6}+2 \mathrm{e}^{2 \varepsilon}\left(B_{5}\left[1+\left(\gamma^{-}\right)^{2}\right]-4 \gamma^{-} \varepsilon^{2}\right)\right]\left(\mu^{+}\right)^{2}\right],
\end{aligned}
$$


with

$$
\begin{array}{lll}
B_{1}^{ \pm}=\gamma^{ \pm}-1, & B_{6}=1+\mathrm{e}^{4 \varepsilon}, & B_{11}^{ \pm}=B_{3}^{ \pm} \mp B_{15}^{ \pm}, \\
B_{2}^{ \pm}=\gamma^{ \pm}+1, & B_{7}=\mu^{-}-\mu^{+}, & B_{12}=B_{2}^{+} \mu^{-}+B_{1}^{+} \mu^{+}, \\
B_{3}^{ \pm}=1 \pm \varepsilon, & B_{8}^{ \pm}=1 \pm \gamma^{-} \gamma^{+}, & B_{13}^{ \pm}=\mathrm{e}^{2 \varepsilon}\left(B_{4}^{+}+2 \varepsilon^{2}\right) \pm 1, \\
B_{4}^{ \pm}=1 \pm 2 \varepsilon, & B_{9}^{ \pm}=\mathrm{e}^{2 \varepsilon} \pm B_{4}^{ \pm}, & B_{14}^{ \pm}=\mathrm{e}^{2 \varepsilon}\left(4 \varepsilon^{2} \pm B_{4}^{ \pm}\right)-1, \\
B_{5}=1+2 \varepsilon^{2}, & B_{10}^{ \pm}=B_{9}^{ \pm}-4 \varepsilon^{2}, & B_{15}^{ \pm}=\gamma^{-}\left(\varepsilon-\gamma^{+} B_{3}^{ \pm}\right) \pm \gamma^{+} \varepsilon .
\end{array}
$$

In this case, stress components can be found, substituting displacements (78) with (80)-(84) into the corresponding expressions in (6) and (7).

\section{Appendix 2}

Consider a homogeneous elastic half-space $\left(\xi_{3}^{+} \geq 0\right)$ subject to the boundary conditions presented in Table 2, where $P=A \mu^{-} \cos \xi_{1}$ is a vertical harmonic load.

\begin{tabular}{|c|c|c|c|c|}
\hline & Case 1 & Case 2 & Case 3 & Case 4 \\
\hline \multicolumn{5}{|c|}{ Boundary conditions for a homogeneous half-space } \\
\hline$\sigma_{33}^{+}$ & $\left.\frac{\kappa \mu^{+}}{12 a} \frac{\partial^{4} u_{3}^{+}}{\partial \xi_{1}^{4}}\right|_{\xi_{3}^{+}=0}-P$ & $-P$ & $-P$ & $-P$ \\
\hline$u_{1}^{+}$ & 0 & 0 & - & - \\
\hline$\sigma_{13}^{+}$ & - & - & $-\left.\frac{\kappa \mu^{+}}{a} \frac{\partial^{2} u_{1}^{+}}{\partial \xi_{1}^{2}}\right|_{\xi_{3}^{+}=0}$ & 0 \\
\hline \multicolumn{5}{|c|}{ Coefficients in $(80)_{3}$ and $(80)_{4}$} \\
\hline$\frac{c_{5} \mu}{A a}$ & $\frac{3 \gamma^{-}\left(\gamma^{+}+1\right)}{\gamma^{-}-1-\gamma^{+}+7 \gamma^{-} \gamma^{+}}$ & $\frac{\gamma^{+}+1}{2 \gamma^{+}}$ & $\frac{\gamma^{-}\left(2+3 \gamma^{+}\right)-2\left(1+\gamma^{+}\right)}{2\left[\gamma^{-}\left(3 \gamma^{+}-1\right)-2 \gamma^{+}\right]}$ & $\frac{\gamma^{+}}{2\left(\gamma^{+}-1\right)}$ \\
\hline$\frac{c_{6} \mu}{A a}$ & $\frac{3 \gamma^{-}\left(\gamma^{+}-1\right)}{\gamma^{-}-1-\gamma^{+}+7 \gamma^{-} \gamma^{+}}$ & $\frac{\gamma^{+}-1}{2 \gamma^{+}}$ & $\frac{\left(3 \gamma^{-}-2\right)\left(\gamma^{+}-1\right)}{2\left[\gamma^{-}\left(3 \gamma^{+}-1\right)-2 \gamma^{+}\right]}$ & $\frac{1}{2}$ \\
\hline \multicolumn{5}{|c|}{ Displacement and stress components at the surface $\xi_{3}^{+}=0$} \\
\hline$\frac{u_{1}^{+} \mu}{A a \sin \xi_{1}}$ & - & - & $\frac{\gamma^{-}}{2\left(\gamma^{-}+2 \gamma^{+}-3 \gamma^{-} \gamma^{+}\right)}$ & $\frac{1}{2\left(\gamma^{+}-1\right)}$ \\
\hline$\frac{u_{3}^{+} \mu}{A a \cos \xi_{1}}$ & $\frac{3 \gamma^{-}\left(\gamma^{+}+1\right)}{\gamma^{-}-1-\gamma^{+}+7 \gamma^{-} \gamma^{+}}$ & $\frac{\gamma^{+}+1}{2 \gamma^{+}}$ & $\frac{\gamma^{-}\left(2+3 \gamma^{+}\right)-2\left(1+\gamma^{+}\right)}{2\left[\gamma^{-}\left(3 \gamma^{+}-1\right)-2 \gamma^{+}\right]}$ & $\frac{\gamma^{+}}{2\left(\gamma^{+}-1\right)}$ \\
\hline$\frac{\sigma_{13}^{+}}{A \mu^{-} \sin \xi_{1}}$ & $\frac{6 \gamma^{-}}{1+\gamma^{+}-\gamma^{-}\left(1+7 \gamma^{+}\right)}$ & $-\frac{1}{\gamma^{+}}$ & $-\frac{2\left(\gamma^{-}-1\right)}{\gamma^{-}\left(3 \gamma^{+}-1\right)-2 \gamma^{+}}$ & - \\
\hline$\frac{\sigma_{33}^{+}}{A \mu^{-} \cos \xi_{1}}$ & $\frac{6 \gamma^{-} \gamma^{+}}{1+\gamma^{+}-\gamma^{-}\left(1+7 \gamma^{+}\right)}$ & - & - & - \\
\hline
\end{tabular}

The equations of the formulated plane strain problem and the solution are given by $(10)_{3},(10)_{4}$ and (78) with functions $(80)_{3}$ and $(80)_{4}$, where $c_{0}^{+}$is taken as $(81)_{1}$, and the values of the coefficients $c_{5}$ and $c_{6}$, corresponding to the appropriate boundary conditions, are presented in Table 2, together with the rest of the displacement and stress components at the surface $\xi_{3}^{+}=0$.

TABLE 2. BVPs for a homogeneous half-space 


\section{References}

[1] Achenbach, J.: Wave propagation in elastic solids. Elsevier (2012)

[2] Aghalovyan, L.: Asymptotic theory of anisotropic plates and shells. World Scientific (2015)

[3] Aleksandrova, G.P.: Contact problems in bending of a slab lying on an elastic foundation. Izv. Akad. Nauk SSSR, Mekh. Tverd. Tela (1) (1973)

[4] Alexandrov, V.M.: Contact problems on soft and rigid coatings of an elastic half-plane. Mech. Solids 45(1), 34-40 (2010)

[5] Bigoni, D., Gei, M., Movchan, A.B.: Dynamics of a prestressed stiff layer on an elastic half space: filtering and band gap characteristics of periodic structural models derived from long-wave asymptotics. J. Mech. Phys. Solids 56(7), 2494-2520 (2008)

[6] Bigoni, D., Ortiz, M., Needleman, A.: Effect of interfacial compliance on bifurcation of a layer bonded to a substrate. Int. J. Solids Struct. 34(33-34), 4305-4326 (1997)

[7] Biot, M.A.: Bending of an infinite beam on an elastic foundation. Z. Angew. Math. Phys. 2(3), 165-184 (1922)

[8] Cai, Z., Fu, Y.: Exact and asymptotic stability analyses of a coated elastic half-space. Int. J. Solids Struct. 37(22), 3101-3119 (2000)

[9] Cai, Z., Fu, Y.: On the imperfection sensitivity of a coated elastic half-space. Proc. R. Soc. A 455(1989), 3285-3309 (1999)

[10] Dai, H.-H., Kaplunov, J., Prikazchikov, D.A.: A long-wave model for the surface elastic wave in a coated half-space. Proc. R. Soc. A 466(2122), 3097-3116 (2010)

[11] Den Hartog, J.P.: Advanced Strength of Materials. McGraw-Hill, Dover, New York (1952)

[12] Destrade, M., Fu, Y., Nobili, A.: Edge wrinkling in elastically supported pre-stressed incompressible isotropic plates. Proc. R. Soc. A 472(2193), 20160410 (2016)

[13] Erbaş, B., Yusufoğlu, E., Kaplunov, J.: A plane contact problem for an elastic orthotropic strip. J. Eng. Math. 70(4), 399-409 (2011)

[14] Fu, Y.B., Cai, Z.X.: An asymptotic analysis of the period-doubling secondary bifurcation in a film/substrate bilayer. SIAM J. Appl. Math. 75(6), 2381-2395 (2015)

[15] Gei, M., Ogden, R.W.: Vibration of a surface-coated elastic block subject to bending. Math. Mech. Solids 7(6), 607-628 (2002)

[16] Goldenveizer, A.L., Kaplunov, J.D., Nolde, E.V.: On Timoshenko-Reissner type theories of plates and shells. Int. J. Solids Struct. 30(5), 675-694 (1993)

[17] Gorbunov-Posadov, M.I.: Beams and Plates on Elastic Foundation [in Russian]. Gosstroiizdat (1949)

[18] Gorbunov-Posadov, M.I.: Calculation of Constructions on Elastic Foundation [in Russian]. Gosstroiizdat, Moscow (1953)

[19] Gorbunov-Posadov, M.I.: Tables for the Computation of Thin Plates on Elastic Foundations [in Russian]. Gosstroiizdat (1959)

[20] Hetenyi, M.: Beams on Elastic Foundation. The University of Michigan Press, Ann Arbor (1958)

[21] Kaplunov, JD: Long-wave vibrations of a thinwalled body with fixed faces. Q. J. Mech. Appl. Math. 48(3), 311-327 (1995)

[22] Kaplunov, JD and Markushevich, DG: Plane vibrations and radiation of an elastic layer lying on a liquid half-space. Wave Motion 17(3), 199-211 (1993)

[23] Kaplunov, JD and Nolde, EV: Long-Wave Vibrations of a Nearly Incompressible Isotropic Plate with Fixed Faces. Q. J. Mech. Appl. Math. 55(3), 345-356 (2002)

[24] Kaplunov, J., Prikazchikov, D., Sultanova, L.: Justification and refinement of Winkler-Fuss hypothesis. Z. Angew. Math. Phys. 69(3), 80 (2018)

[25] Kuznetsov, V.I.: Elastic Foundations. Gosstroiizdat, Moscow (1952)

[26] Popov, G. Ya.: Plates on a linearly elastic foundation (a survey). Soviet Applied Mechanics 8(3), 231-242 (1972)

[27] Steigmann, D.J., Ogden, R.W.: Plane deformations of elastic solids with intrinsic boundary elasticity. Proc. R. Soc. A 453(1959), 853-877 (1997)

[28] Tiersten, H.F.: Elastic surface waves guided by thin films. J. Appl. Phys. 40(2), 770-789 (1969) 
J. Kaplunov, D. Prikazchikov, L. Sultanova

School of Computing and Mathematics, Keele University, Keele, Staffordshire, ST55BG, UK

e-mail: j.kaplunov@keele.ac.uk, d.prikazchikov@keele.ac.uk, 1.sultanova@keele.ac.uk 